\title{
Successful treatment of two relapsed patients with $t(11 ; 19)(q 23 ; p 13)$ acute myeloid leukemia by CLAE chemotherapy sequential with allogeneic hematopoietic stem cell transplantation: Case reports
}

\author{
SHANDONG TAO ${ }^{1,2}$, LIXIAO SONG ${ }^{1,2}$, YUAN DENG $^{1,2}$, YUE CHEN $^{1,2}$, YIMIN GAN $^{1,2}$, YUNJIE LI $^{1,2}$, \\ YIHAN DING $^{1,2}$, ZHE ZHANG ${ }^{1,2}$, BANGHE DING ${ }^{1,2}$, ZHENGMEI HE ${ }^{1,2}$, CHUNLING WANG $^{1,2}$ and LIANG YU $^{1,2}$ \\ ${ }^{1}$ Department of Hematology, The Affiliated Huai'an No. 1 People's Hospital of Nanjing Medical University, \\ Huai'an, Jiangsu 223300; ${ }^{2}$ Key Laboratory of Hematology, Nanjing Medical University, Nanjing, Jiangsu 210029, P.R. China
}

Received February 28, 2020; Accepted November 20, 2020

DOI: $10.3892 / \mathrm{ol} .2021 .12439$

\begin{abstract}
The prognosis of patients with relapsed/refractory acute myeloid leukemia (R/R AML) is poor, with a 3-year overall survival rate of $10 \%$. Patients with translocation $(\mathrm{t})$ $(11 ; 19)(q 23 ; p 13)$ have a higher risk of relapse and there is no optimal regimen for these patients. The present study treated two young patients with $\mathrm{t}(11 ; 19)(\mathrm{q} 23 ; \mathrm{p} 13)$ AML, who relapsed after one or two cycles of consolidation, with a salvage treatment consisting of sequential cladribine, cytarabine and etoposide (CLAE) and allogeneic hematopoietic stem cell transplantation (allo-HSCT). Both neutrophil and platelet engraftments were achieved within 15 days, and no severe transplant-related complications and graft-versus-host diseases were observed. Following allo-HSCT, both patients achieved complete hematologic and cytogenetic remission. Decitabine was used for the prophylaxis of relapse. The two patients remained alive and disease-free for 100 days following allo-HSCT. The results presented here suggest that CLAE regimen sequential with allo-HSCT may be effective in treating patients with $\mathrm{R} / \mathrm{R}$ AML, with $\mathrm{t}(11 ; 19)(\mathrm{q} 23 ; \mathrm{p} 13)$. However, further studies and a larger sample size are required to validate the effectiveness of this treatment regimen.
\end{abstract}

Correspondence to: Professor Chunling Wang or Professor Liang Yu, Department of Hematology, The Affiliated Huai'an No. 1 People's Hospital of Nanjing Medical University, 1 Huang River Road West, Huai'an, Jiangsu 223300, P.R. China

E-mail:wc16506@163.com

E-mail: yuliangha@163.com

Abbreviations: $\mathrm{R} / \mathrm{R}$ AML, relapsed/refractory acute myeloid leukemia; CLAE, cladribine, cytarabine and etoposide; allo-HSCT, allogeneic hematopoietic stem cell transplantation; GVHD, graft-versus-host disease; CR, complete remission; MRD, minimal residual disease; OS, overall survival

Key words: CLAE, relapse, acute myeloid leukemia, translocation $(11 ; 19)$ (q23;p13), allogeneic hematopoietic stem cell transplantation

\section{Introduction}

Acute myeloid leukemia (AML) is a malignant clonal disorder that originates from hematopoietic stem cells by uncontrolled proliferation and suppressed differentiation of blast cells in the myeloid lineage, which infiltrate the bone marrow, blood and other tissues, including lymph node or spleen (1). AML is the most common type of acute leukemia in adults, which accounts for $1.3 \%$ of new cancer cases annually in the United states (2). AML can occur in any age group; however, the most affected patients are older adults, with a median age at diagnosis of 68 years (2). It is estimated that $10-40 \%$ of patients newly diagnosed with AML fail to achieve complete remission (CR) with the standard induction chemotherapy treatment, and these cases are defined as primary refractory cases (3). In addition, most patients who achieve CR will eventually relapse because of unfavorable cytogenetics at diagnosis and old age $(4,5)$. Patients with relapsed/refractory $(\mathrm{R} / \mathrm{R}) \mathrm{AML}$ have a poor prognosis, with a 3-year overall survival (OS) rate of only $10 \%$, thus the majority of patients die from recurrence $(5,6)$.

Currently, there is no standard treatment regimen for patients with R/R AML, except for allogeneic hematopoietic stem cell transplantation (allo-HSCT) (6,7). Most R/R AML patients are old and have less than ideal performance status or without a matched related donor, so only a minority of patients are suitable for allo-HSCT (5). Thus, it remains critical to identify early prognostic factors and select the appropriate conditioning regimens for allo-HSCT for the effective treatment of patients with AML.

Cladribine is a deoxyadenosine analogue that can be rapidly phosphorylated into a triphosphate form, which resists degradation by adenosine deaminase and increases cytotoxic levels in the intracellular space, inhibits DNA synthesis and induces cell apoptosis (8). Previous studies have demonstrated that cladribine combination regimens are effective in patients with $\mathrm{R} / \mathrm{R}$ AML and may overcome abnormal chromosome karyotypes with poor prognosis $(8,9)$. In addition, cladribine-based chemotherapy regimens sequential with allo-HSCT are considered promising treatment strategies for patients with R/R AML $(10,11)$. 
Conventional cytogenetic data has established prognostic indications for patients with AML (2). Based on karyotypic analysis, recurring translocations (t) or inversions (inv) or deletion (del), including $\mathrm{t}(6 ; 9), \mathrm{t}(\mathrm{v} .11 \mathrm{q} 23.3), \mathrm{t}(9 ; 22)$, inv(3), $\mathrm{t}(3 ; 3),-5$, del $(5 q),-7$ and -17 are associated with adverse risk of AML, designated by the World Health Organization (2). $\mathrm{t}(11 ; 19)(\mathrm{q} 23 ; \mathrm{p} 13)$ is a rare recurrent cytogenetic abnormality in patients with AML; however, its clinical and genetic characteristics are not yet fully understood (12). Previous studies have reported that the prognosis of patients with AML, with $t(11 ; 19)$ (q23;p13) is poor, with a median OS time $<1$ year $(13,14)$. It has been reported that CLAM (cladribine, cytarabine, mitoxantrone) and MEC (mitoxantrone, etoposide, and cytarabine) chemotherapy regimens exhibit promising activity in patients with R/R AML $(15,16)$, thus, CLAM was selected as the regimen in the present study. The present study reported two relapsed patients with $\mathrm{t}(11 ; 19)(\mathrm{q} 23 ; \mathrm{p} 13)$ AML who were successfully treated with cladribine, cytarabine and etoposide (CLAE) chemotherapy sequential with allo-HSCT.

\section{Case reports}

The present study was approved by the Institutional Review Board of The Affiliated Huai'an No. 1 People's Hospital of Nanjing Medical University (Huai'an, China; approval no. YX-P-2020-004-01) and performed in accordance with the Declaration of Helsinki (17). Written informed consent was provided by both patients prior to the study start.

Case 1. On December 26, 2018, a 23-year-old man was admitted to The Affiliated Huai'an No. 1 People's Hospital of Nanjing Medical University due to stomachaches and experiencing weakness. Peripheral blood analysis revealed the following: White blood cell count of $70.85 \times 10^{9} / 1$, hemoglobin level of $114 \mathrm{~g} / 1$ and a platelet count of $26 \times 10^{9} / 1$. In addition, the bone marrow (BM) smear revealed $42 \%$ of blast cells. Immunophenotype analysis indicated that $33 \%$ of the blast cells were abnormal, and demonstrated positive labeling for myeloperoxidase (MPO), cluster of differentiation (CD)13, CD33, CD34, CD38 and CD117. The karyotype was 46,XY,t(11;19) (q23;p13)[17]/46,XY[3] (Fig. 1). Aberrant EVII and NRAS expression levels were detected (mutation frequency, 8.32\%) via reverse transcription (RT)-PCR and sanger sequencing analyses, while fusion genes were detected via RT-PCR analysis. Total RNA was extracted using the Omega whole-blood RNA extraction kit (cat. no. R6616-02; Omega Bio-Tek, Inc.), according to the manufacturer's protocol. Nested RT-PCR analysis was performed, and the reaction system was used as previously described $(18,19)$. AML-associated mutated genes were detected using high-throughput sequencing technology. Total DNA was extracted using the whole blood DNA extraction kit (cat. no. D3392-02; Omega Bio-Tek, Inc.), and genetic mutations were detected by the Kindstar Global Medical Laboratory Center (http://www.kindstar.com. $\mathrm{cn} / \mathrm{kindstar} / \mathrm{cn} /$ platform.html), using sanger sequencing as previously described $(19,20)$. Notably, $M L L$ rearrangement was not detected. The patient was diagnosed with AML (M4) according to the FAB (French American British) classification system (21). The patient was treated with standard ' $3+7$ ' regimen with idarubicin and cytarabine (IA) as induction therapy and achieved CR, with $1.5 \%$ blasts after one cycle. The minimal residual disease (MRD) detection via flow cytometric $(\mathrm{FCM})$ analysis was negative $(<0.01 \%)$ and was followed by three cycles of consolidation (intermediate dose cytarabine with $2.0 \mathrm{~g} / \mathrm{m}^{2} \times 6$ times). Following the third consolidation chemotherapy, MRD detection was positive, with 6\% abnormal cells expressing CD13, CD33 and CD34 via FCM analysis, and the BM smear indicated relapse ( $8.5 \%$ of blast cells; Fig. 2). The patient received one cycle of homoharringtonine, daunorubicin and cytarabine reinduction chemotherapy regimen; however, he failed to achieve CR. The BM blasts were $11.5 \%$ and the MRD was $10.5 \%$. The patient subsequently received intense CLAE chemotherapy (cladribine $5 \mathrm{mg} / \mathrm{m}^{2} / \mathrm{dx} 5 \mathrm{~d}$, cytarabine $1.5 \mathrm{~g} / \mathrm{m}^{2} / \mathrm{dx} 5 \mathrm{~d}$ and etoposide $100 \mathrm{mg} / \mathrm{m}^{2} / \mathrm{dx} 3 \mathrm{~d}$ ), and underwent haploid allo-HSCT (stem cells from his father) 3 days after CLAE chemotherapy, with the conditioning regimen of Flu-Bu-ATG (fludarabine $30 \mathrm{mg} / \mathrm{m}^{2} / \mathrm{dx} 5 \mathrm{~d}$, busulfan $3.2 \mathrm{mg} / \mathrm{kg} / \mathrm{dx} 3 \mathrm{~d}$ and ATG $2.5 \mathrm{mg} / \mathrm{kg} / \mathrm{dx} 4 \mathrm{~d}$ ). Graft-versus-host diseases (GVHDs) were prevented by cyclosporin A, methotrexate and mycophenolate mofetil (Fig. 3). A total of $12.5 \times 10^{8} / \mathrm{kg}$ mononuclear cells and $2.75 \times 10^{6} / \mathrm{kg} \mathrm{CD} 34^{+}$cells from the donor were intravenously injected into the patient, and no serious complications occurred during allo-HSCT. The neutrophil and platelet engraftments were achieved on days 14 and 12, respectively (Fig. 4C and D). The MRD was $10^{-4}$ as detected via FCM analysis, and the chromosome changed to normal karyotype 1 month after transplantation. The donor chimerism rate was $100 \%$ following multiplex PCR analysis of short tandem repeats at +14 days, +30 days and +60 days after transplantation. The decitabine regimen (decitabine $15 \mathrm{mg} / \mathrm{m}^{2} / \mathrm{dx} 5 \mathrm{~d}$ ) was implemented every 3 months for the prophylaxis of relapse, beginning at 2 months after allo-HSCT. The patient received follow-up in the clinic weekly and remained in a disease-free survival state based on the last follow-up at 4.3 months after allo-HSCT.

Case 2. On May 12, 2019, a 31-year-old man was admitted to The Affiliated Huai'an No. 1 People's Hospital of Nanjing Medical University due to fever and bleeding gums. Peripheral blood analysis revealed the following: White blood cell count of $10.37 \times 10^{9} / 1$, hemoglobin level of $114 \mathrm{~g} / 1$ and a platelet count of $8 \times 10^{9} / 1$. In addition, the BM smear revealed $28 \%$ of blasts cells. FCM analysis exhibited $39 \%$ of blast cells, and demonstrated positive labeling for MPO, CD13, CD15, CD33, CD38, CD56 and human leukocyte antigen-DR (HLA-DR). The karyotype was 46,XY,t(11;19)(q23;p13.1)[18]/46,XY[2] (Fig. 1). Aberrant EVII and $M L L / E L L$ rearrangement were detected using the same methods performed for patient 1 . The patient was diagnosed with AML (M2) according to the FAB classification and was subsequently treated with two cycles of IA regimen as induction, and achieved CR with $2.0 \%$ blasts. The MRD was negative $(<0.01 \%)$ and was followed by one cycle of consolidation with an intermediate dose of cytarabine with $2.0 \mathrm{~g} / \mathrm{m}^{2}$ x 6 times. The patient was re-admitted due to severe crissum infection on October 10, 2019. The BM smear revealed $58 \%$ of blast cells 6 months after consolidation, suggesting disease relapse (Fig. 2). The patient underwent matched unrelated allo-HSCT (stem cells from a donor at the Chinese bone marrow bank) 
Patient 1

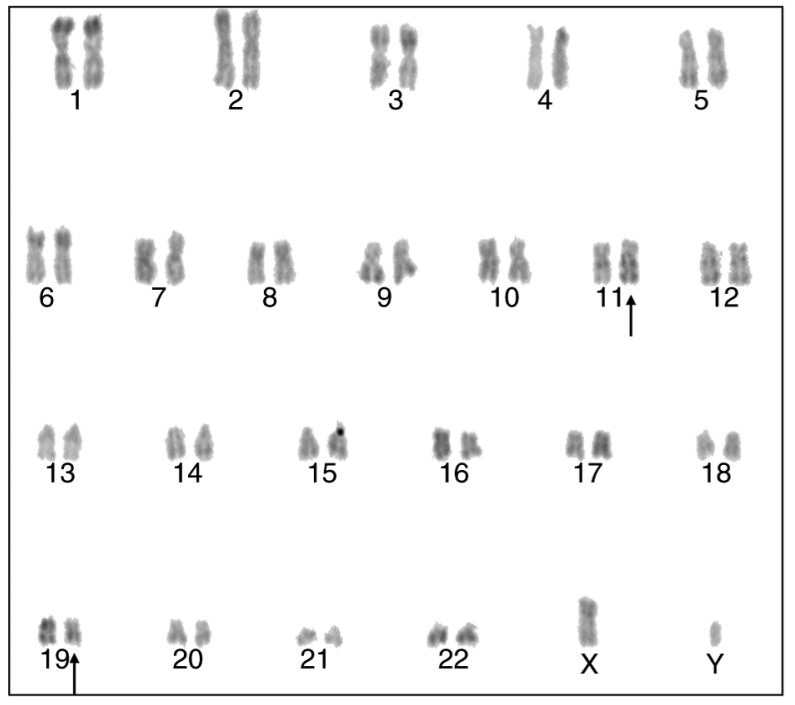

Patient 2

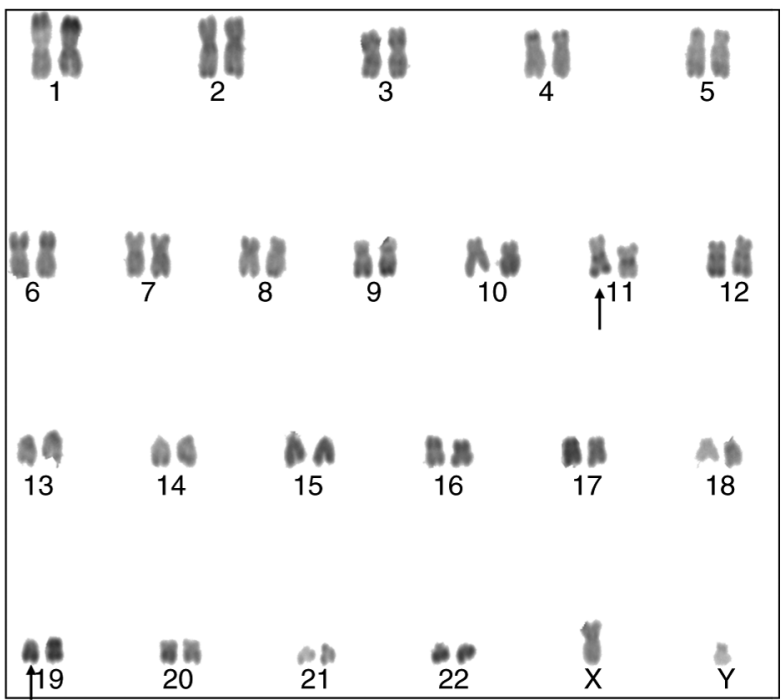

Figure 1. Chromosome karyotype of two t(11;19) patients with AML. G-banded karyotype of BM cells at the newly diagnosed stage. Patient 1: Representative images of the karyotype of BM cells with $\mathrm{t}(11 ; 19)(\mathrm{q} 23 ; \mathrm{p} 13)$. Patient 2 : Representative images of the karyotype of BM cells with $\mathrm{t}(11 ; 19)(\mathrm{q} 23 ; \mathrm{p} 13.1)$. The arrows indicate translocated chromosomes. t, translocation; AML, acute myeloid leukemia; BM, bone marrow.

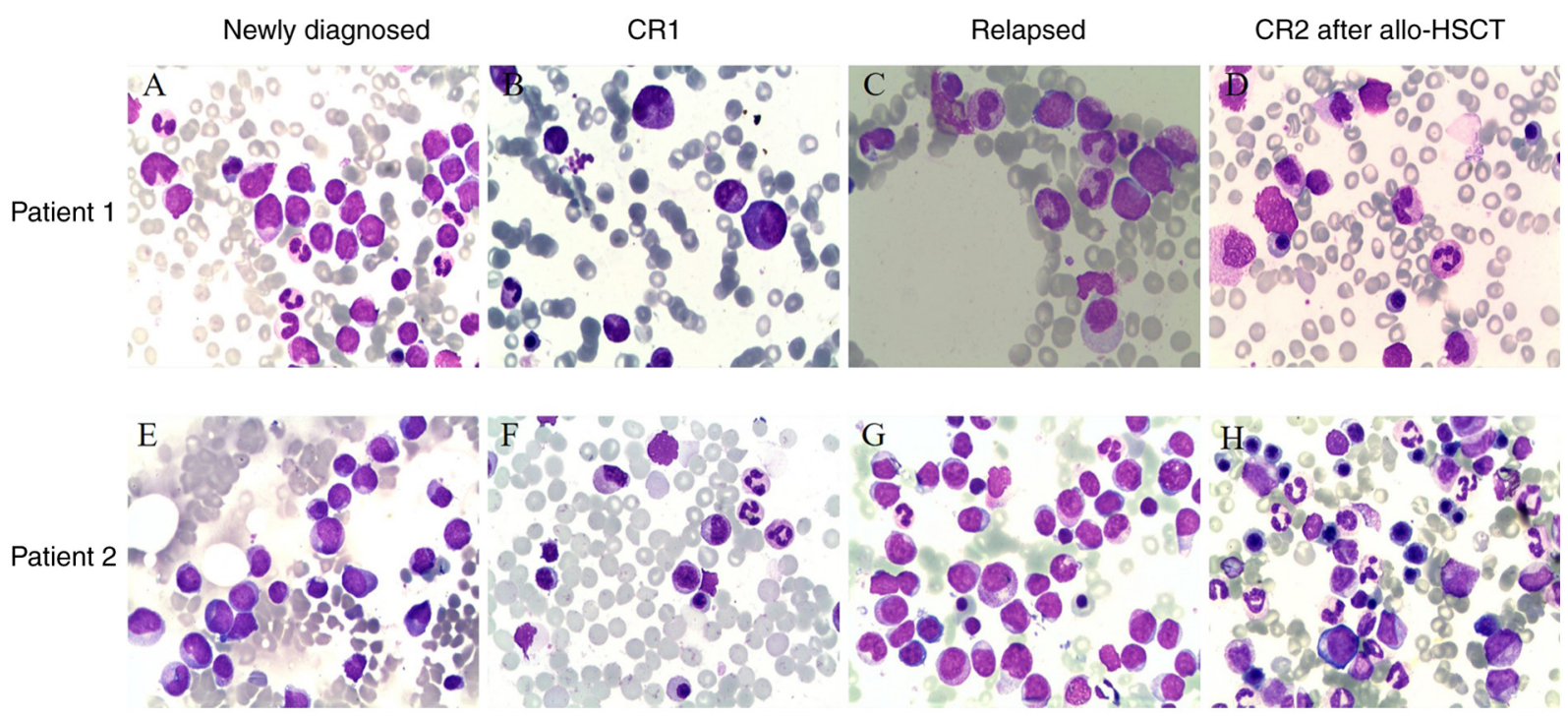

Figure 2. Morphological features of two patients with AML at different stages of the disease. May-Grüwald-Giemsa-stained BM smear (magnification, $\mathrm{x} 1,000$ ), Patient 1: (A) Morphological features of BM at the newly diagnosed stage, granulocytes increased and myeloblasts accounted for $42 \%$. The positive rate of myeloperoxidase staining was 76\%. (B) Morphological features following induction chemotherapy. (C) Morphological features at the relapsed stage, myeloblasts accounted for 9\%. (D) Morphological features following allo-HSCT. Patient 2: (E) Morphological features of BM at the newly diagnosed stage, granulocytes increased and myeloblasts accounted for $28 \%$. The positive rate of myeloperoxidase staining was $92 \%$. (F) Morphological features following induction chemotherapy. (G) Morphological features at the relapsed stage, myeloblasts accounted for 58\%. (H) Morphological features following allo-HSCT. AML, acute myeloid leukemia; BM, bone marrow; allo-HSCT, allogeneic hematopoietic stem cell transplantation; CR, complete remission.

and simultaneously received antibiotics to control the crissum infection. The patient received intense CLAE chemotherapy (cladribine $5 \mathrm{mg} / \mathrm{m}^{2} / \mathrm{dx} 5 \mathrm{~d}$, cytarabine $1.5 \mathrm{~g} / \mathrm{m}^{2} / \mathrm{dx} 5 \mathrm{~d}$ and etoposide $100 \mathrm{mg} / \mathrm{m}^{2} / \mathrm{dx} 3 \mathrm{~d}$ ) and conditioning regimen, followed by allo-HSCT. The BM smear revealed $23 \%$ of blasts cells following CLAE, thus the total dose of busulfan in the conditioning regimen was adjusted to $3.2 \mathrm{mg} / \mathrm{kg} / \mathrm{dx} 4 \mathrm{~d}$. The conditioning regimen consisted of the following: Fludarabine $30 \mathrm{mg} / \mathrm{m}^{2} / \mathrm{x} 5 \mathrm{~d}$, busulfan $3.2 \mathrm{mg} / \mathrm{kg} / \mathrm{dx} 4 \mathrm{~d}$ and ATG $2.5 \mathrm{mg} / \mathrm{kg} / \mathrm{dx} 4 \mathrm{~d}$. Cyclosporin A, methotrexate and mycophenolate mofetil were used to prevent GVHDs. A total of
$8.0 \times 10^{8} / \mathrm{kg}$ mononuclear cells and $6.72 \times 10^{6} / \mathrm{kg} \mathrm{CD} 34^{+}$cells were intravenously infused into the patient. The patient suffered crissum abscess and sepsis during transplantation. The neutrophil and platelet engraftments were achieved on days 12 and 14, respectively (Fig. 4C and D). MRD detection was negative $\left(<10^{-4}\right)$, and the chromosome changed to normal karyotype 1 month after transplantation. The donor chimerism rate was $100 \%$ at +14 days, +30 days and +60 days following transplantation. The decitabine regimen was implemented for the prophylaxis of relapse. The patient received follow-up in the clinic weekly and achieved CR based on the last follow-up 


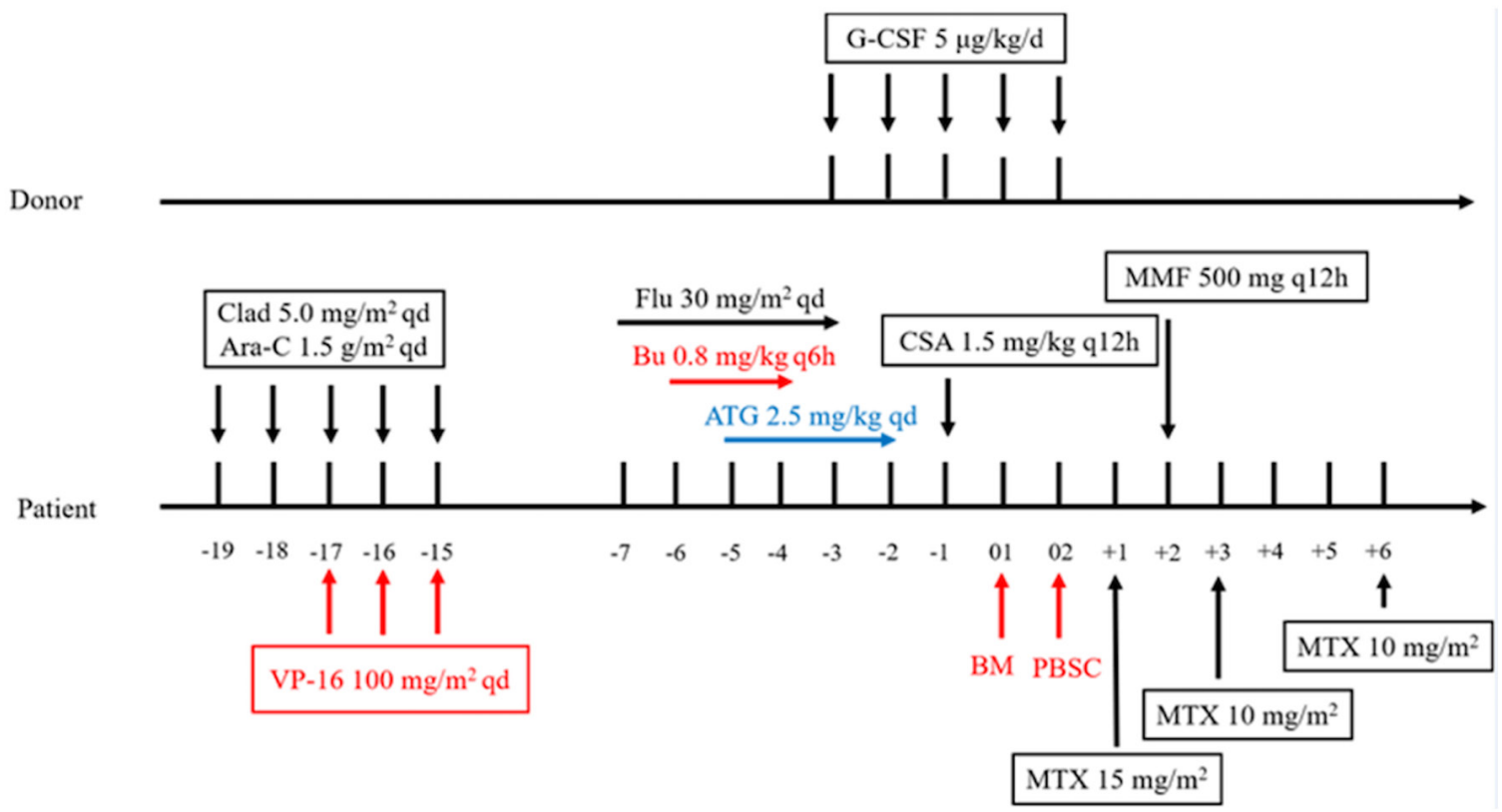

Figure 3. Flowchart of regimen consisting of cladribine, cytarabine and etoposide chemotherapy, and allogeneic hematopoietic stem cell transplantation. Clad, cladribine; Ara-C, cytarabine; VP-16, etoposide; Flu, fludarabine; Bu, busulfan; ATG, anti-thymocyte globulin; G-CSF, granulocyte colony-stimulating factor; CSA, cyclosporin A; BM, bone marrow; PBSC, peripheral blood stem cell; MTX, methotrexate; MMF, mycophenolate mofetil.
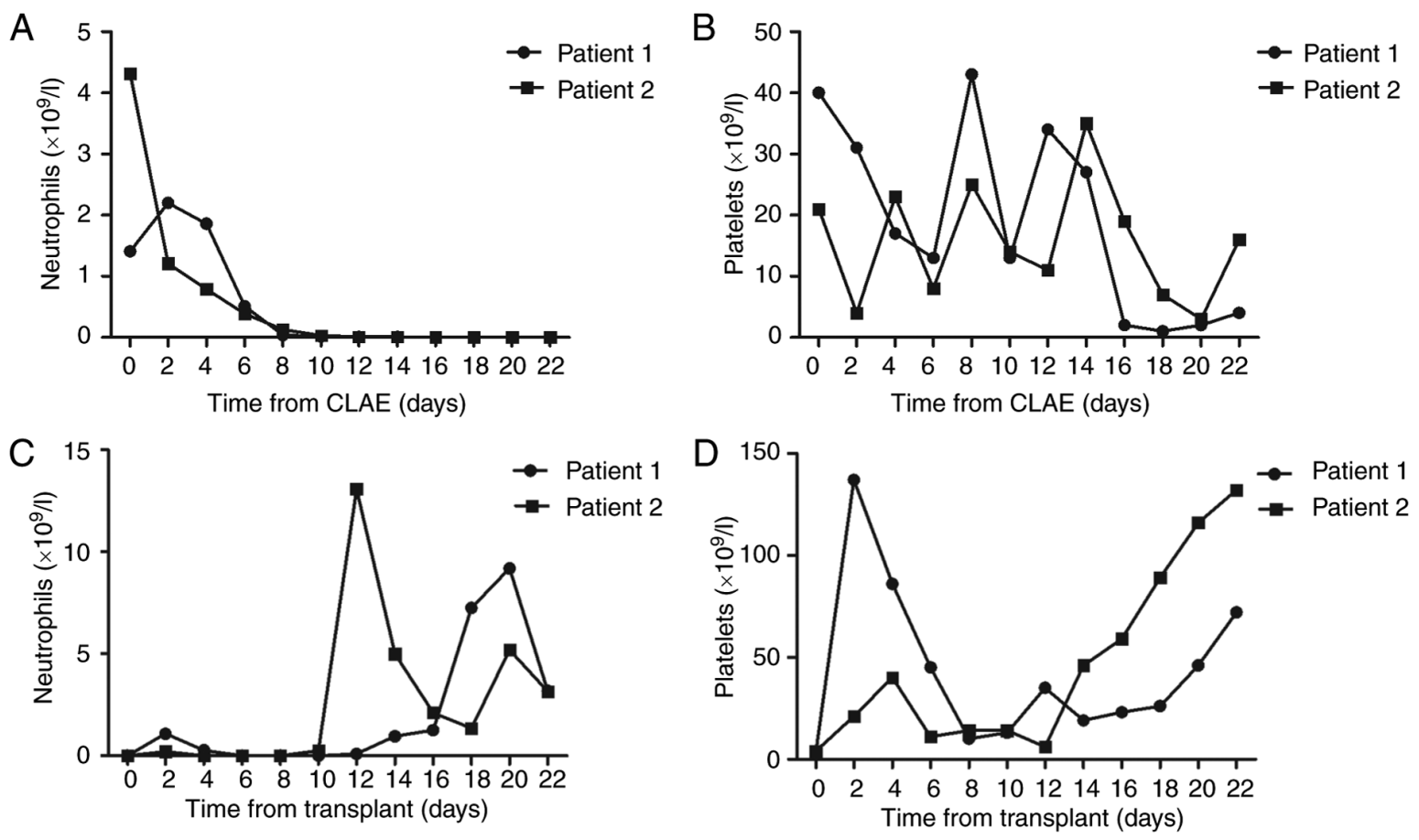

Figure 4. Recovery of platelets and neutrophils following allo-HSCT. (A and B) The recovery times achieved a normal neutrophil count following CLAE regimen of patient 1 and patient 2. (C and D) The recovery times achieved a normal neutrophil count following allo-HSCT of patient 1 and patient 2 . allo-HSCT, allogeneic hematopoietic stem cell transplantation; CLAE, regimen consisting of cladribine, cytarabine and etoposide.

at 3.5 months after allo-HSCT. The characteristics of both patients are presented in Table I.

\section{Discussion}

In the present study, two relapsed patients with $t(11 ; 19)$ (q23;p13) AML were successfully treated with CLAE regimens sequential with allo-HSCT. Both patients achieved CR.
The intense chemotherapy prior to allo-HSCT decreased the leukemia burden and inhibited the immune system of the recipient to promote the implantation of HSCs. The results presented here confirm the efficacy of intense CLAE chemotherapy sequential with allo-HSCT in young relapsed patients with $\mathrm{t}(11 ; 19)(\mathrm{q} 23 ; \mathrm{p} 13)$ AML. Hematological toxicity and other side effects, including hemocytopenia, nausea and vomiting, infection were presented during treatment. 
Table I. Patient characteristics.

\begin{tabular}{|c|c|c|}
\hline Characteristic & Case 1 & Case 2 \\
\hline Age, year & 23 & 31 \\
\hline Sex & Male & Male \\
\hline WBC, $\times 10^{9} / 1$ & 70.85 & 10.37 \\
\hline PLT, $x 10^{9} / 1$ & 26 & 8 \\
\hline Blasts in bone marrow, $\%$ & 42 & 39 \\
\hline FAB & AML-M4 & AML-M2 \\
\hline Cytogenetics & 46,XY,t(11;19)(q23;p13)[17]/46,XY[3] & 46,XY,t(11;19)(q23;p13.1)[18]/46,XY[2] \\
\hline Molecular biology & EVI1, NRAS & EVI1, MLL/ELL \\
\hline Risk stratification & High risk & High risk \\
\hline Induction chemotherapy regimen & IA & IA \\
\hline MRD after induction chemotherapy & Negative & Negative \\
\hline Disease status before HSCT & Relapsed & Relapsed \\
\hline
\end{tabular}

WBC, white blood cells; PLT, platelet; FAB, French American British; MRD, minimal residual disease, HSCT, hematopoietic stem cell transplantation, IA, idarubicin and cytarabine.

Reciprocal chromosomal translocations can cause genetic aberrations in pediatric and adult patients with AML $(22,23)$. $\mathrm{t}(11 ; 19)(\mathrm{q} 23 ; \mathrm{p} 13)$ is a relatively rare recurrent cytogenetic aberration that occurs in patients with AML (12). MLL is involved in the majority of 11q23 translocations of acute leukemias, whereby rearrangement of $M L L$ results in fusion of the $M L L$ gene with its partner gene (12). However, heterogeneity rearrangements have been observed at this chromosomal region $(12,24)$. NRAS and $K R A S$ are frequently mutated in $M L L$-rearranged leukemia (25). The present study assessed two patients with $\mathrm{t}(11 ; 19)(\mathrm{q} 23 ; \mathrm{p} 13)$ AML, patient 1 (M4) had no MLL rearrangement, while patient 2 (M2) had MLL/ELL rearrangement, and both presented with early relapse (less than half a year). Consistent with previous findings (12-14), the results of the present study demonstrated that $\mathrm{t}(11 ; 19)(\mathrm{q} 23$;p13) was associated with a poor prognosis and short OS time in patients with AML, and indicated that it is necessary for these patients to receive allo-HSCT in first CR.

The present study is not without limitations. First, the $M L L$ rearrangement in patient 2 was not detected via fluorescence in situ hybridization as the sample size was too small. Secondly, both patients had a relatively short follow-up period, thus further studies are required with extended follow-up periods and larger sample sizes.

Intensive chemotherapy is used to eliminate leukemia cells, followed by allo-HSCT as consolidation therapy for patients with R/R AML (26). This regimen has been demonstrated to improve the long-term survival rate from 20 to $50 \%$; however, reinduction chemotherapy-related side effects must be acceptable to patients (27). Previous studies have reported that FLAG-IDA (granulocyte colony-stimulating factor, fludarabine, cytarabine, and idarubicin), CLAG (cladribine, cytarabine, granulocyte colony-stimulating factor), CLAM (cladribine, cytarabine, mitoxantrone) and MEC (mitoxantrone, etoposide, and cytarabine) chemotherapy regimens achieve high CR rates in patients with R/R AML (28-31). However, CR rates in response to common salvage regimens decrease (10-15\%) in refractory or early relapsed patients with
AML (26). Furthermore, common conditioning regimens followed by allo-HSCT in patients with R/R AML exhibit disappointing results $(32,33)$. Thus, the concept of chemotherapy sequential with allo-HSCT was developed (26). Recently, it has been demonstrated that high-dose melphalan-based sequential conditioning chemotherapy followed by allo-HSCT is feasible in patients with R/R AML (26). Another study reported the feasibility and efficacy of FLAG-IDA chemotherapy sequential with Flu-Bu3 conditioning regimen in patients with refractory AML (34). Based on previous studies (28-31) and patient characteristics, the present study selected the CLAE regimen sequential with Flu-Bu conditioning regimen for allo-HSCT in the assessed patients. Patient 1 received CLAE regimen sequential with haploid transplantation, and exhibited granulocyte deficiency at 21 after chemotherapy, the neutrophil and platelet engraftments were achieved on days 14 and 12. Patient 2 received CLAE regimen sequential with hematopoietic stem cell transplantation from unrelated donors, the neutrophil and platelet engraftments were achieved on days 12 and 14. No serious transplant-related complications occurred in patient 1 , while patient 2 experienced crissum abscess and sepsis, which were effectively controlled with antibiotics. Any grade of GVHDs were absent in both cases. MRD was negative in both patients, and the donor chimerism rate was $100 \%$, while the karyotype of chromosomes changed to normal karyotype. Decitabine was used to prevent disease relapse following transplantation, and both patients remained alive and disease-free based on the last follow-up.

In conclusion, the results of the present study demonstrated the high antileukemic efficacy and acceptable toxicity of CLAE regimen sequential with allo-HSCT for patients with $\mathrm{t}(11 ; 19)$ (q23;p13) AML. The results presented here suggest that $t(11 ; 19)$ (q23;p13) is a poor prognostic factor in AML, and that the CLAE regimen sequential with allo-HSCT may be an effective treatment strategy for patients with $\mathrm{t}(11 ; 19)(\mathrm{q} 23 ; \mathrm{p} 13) \mathrm{R} / \mathrm{R}$ AML. However, prospective studies with larger sample sizes are required to validate the effectiveness of this treatment regimen. 


\section{Acknowledgements}

Not applicable.

\section{Funding}

The present study was supported by research grants from the Science and Technology Fund of Huai'an City (grant no.HAB201810) and the Science and Technology Fund of Jiangsu Commission of Health (grant nos. H2018085 and H2019082).

\section{Availability of data and materials}

The data that support the findings of the present study are available from Kindstar Global Medical Laboratory Center (https://www. kindstar.com.cn/platform.html); however, restrictions apply to the availability of these data, which were used under license for the present study, and so are not publicly available. Data are available from the authors upon reasonable request and with permission from Kindstar Global Medical Laboratory Center.

\section{Authors' contributions}

ST collected patient data and drafted the initial manuscript. CW and LY conceived and designed the present study. ST revised the manuscript along with LS, YD, YC, YG, YL, YHD, $\mathrm{ZZ}, \mathrm{BD}$ and $\mathrm{ZH}$, and these individuals were responsible for the treatment of the patients. All authors have read and approved the final manuscript.

\section{Ethics approval and consent to participate}

The present study was approved by the Institutional Review Board of the Affiliated Huai'an No. 1 People's Hospital of Nanjing Medical University (Huai'an, China; approval no. YX-P-2020-004-01) and performed in accordance with the Declaration of Helsinki. Written informed consent was provided by both patients prior to the study start.

\section{Patient consent for publication}

Both patients provided consent for publication.

\section{Competing interests}

The authors declare that they have no competing interests.

\section{References}

1. Döhner H, Weisdorf DJ and Bloomfield CD: Acute myeloid leukemia. N Engl J Med 373: 1136-1152, 2015.

2. Short NJ, Rytting ME and Cortes JE: Acute myeloid leukaemia. Lancet 392: 593-606, 2018.

3. Thol F, Schlenk RF, Heuser M and Ganser A: How I treat refractory and early relapsed acute myeloid leukemia. Blood 126: 319-327, 2015 .

4. Kurosawa S, Yamaguchi T, Miyawaki S, Uchida N, Sakura T, Kanamori H, Usuki K, Yamashita T, Okoshi Y, Shibayama H, et al: Prognostic factors and outcomes of adult patients with acute myeloid leukemia after first relapse. Haematologica 95: 1857-1864, 2010.

5. Ramos NR, Mo CC, Karp JE and Hourigan CS: Current approaches in the treatment of relapsed and refractory acute myeloid leukemia. J Clin Med 4: 665-695, 2015.
6. Bose P, Vachhani P and Cortes JE: Treatment of relapsed/refractory acute myeloid leukemia. Curr Treat Options Oncol 18: 17, 2017.

7. Yanada M, Mori J, Aoki J, Masuko M, Harada K, Uchida N, Doki N, Fukuda T, Sakura T, Kanamori H, et al: Allogeneic hematopoietic cell transplantation for patients with a history of multiple relapses of acute myeloid leukemia. Ann Hematol 98: 2179-2186, 2019.

8. Robak T and Wierzbowska A: Cladribine in the treatment of acute myeloid leukemia. Leuk Res 38: 425-427, 2014.

9. Fridle C, Medinger M, Wilk MC, Seipel K, Passweg J, Manz MG and Pabst T: Cladribine, cytarabine and idarubicin (CLA-Ida) salvage chemotherapy in relapsed acute myeloid leukemia (AML). Leuk Lymphoma 58: 1068-1075, 2017.

10. Xiao H, Li L, Pang Y, Wu Y, Jiang Z, Liu Z, Wu J, Xiao Y, Huang F, Liu $\mathrm{Q}$, et al: Sequential treatment combining cladribine-based re-induction, myeloablative allogeneic $\mathrm{HSCT}$, and prophylactic donor lymphocyte infusion: A promising treatment for refractory acute myeloid leukemia. Ann Hematol 97: 2479-2490, 2018.

11. Jin M, Hu Y, Wu W, Luo Y, Tan Y, Yu J, Jin A, Yang L, Huang H and Wei G: Decitabine plus CLAG chemotherapy as a bridge to haploidentical transplantation in the setting of acute myeloid leukemia relapse after HLA-matched sibling transplantation: A case report. BMC Cancer 19: 242, 2019.

12. Yamamoto K, Kawamoto S, Kakiuchi S, Yakushijin K, Matsuoka H and Minami H: Translocation t $(11 ; 19)$ (q23; q13.1) without MLL rearrangement in acute myeloid leukemia: Heterogeneity of the 11q23 Breakpoints. Acta Haematol 134: 76-79, 2015

13. Bhatnagar B, Blachly JS, Kohlschmidt J, Eisfeld AK, Volinia S, Nicolet D, Carroll AJ, Block AW, Kolitz JE, Stone RM, et al: Clinical features and gene- and microRNA-expression patterns in adult acute leukemia patients with $\mathrm{t}(11 ; 19)(\mathrm{q} 23 ; \mathrm{p} 13.1)$ and t (11;19) (q23; p13.3). Leukemia 30: 1586-1589, 2016.

14. Chen Y, Kantarjian H, Pierce S, Faderl S, O'Brien S, Qiao W, Abruzzo L, de Lima M, Kebriaei P, Jabbour E, et al: Prognostic significance of 11q23 aberrations in adult acute myeloid leukemia and the role of allogeneic stem cell transplantation. Leukemia 27: 836-842, 2013

15. Wierzbowska A, Robak T, Pluta A, Wawrzyniak E, Cebula B, Hołowiecki J, Kyrcz-Krzemień S, Grosicki S, Giebel S, Skotnicki AB, et al: Cladribine combined with high doses of arabinoside cytosine, mitoxantrone, and G-CSF (CLAG-M) is a highly effective salvage regimen in patients with refractory and relapsed acute myeloid leukemia of the poor risk: A final report of the Polish Adult Leukemia Group. Eur J Haematol 80: 115-126, 2008.

16. Halpern AB, Othus M, Huebner EM, Buckley SA, Pogosova-Agadjanyan EL, Orlowski KF, Scott BL, Becker PS, Hendrie PC, Chen TL, et al: Mitoxantrone, etoposide and cytarabine following epigenetic priming with decitabine in adults with relapsed/refractory acute myeloid leukemia or other high-grade myeloid neoplasms: A phase 1/2 study. Leukemia 31: 2560-2567, 2017.

17. World Medical Association: World Medical Association Declaration of Helsinki: Ethical principles for medical research involving human subjects. JAMA 310: 2191-2194, 2013.

18. Pallisgaard N, Hokland P, Riish øj DC, Pedersen B and Jørgensen P: Multiplex reverse transcription-polymerase chain reaction for simultaneous screening of 29 translocations and chromosomal aberrations in acute leukemia. Blood 92: 574-588, 1998.

19. Yang YL, Lin SR, Chen JS, Hsiao CC, Lin KH, Sheen JM, Cheng CN, Wu KH, Lin SW, Yu SL, et al: Multiplex reverse transcription-polymerase chain reaction as diagnostic molecular screening of 4 common fusion chimeric genes in Taiwanese children with acute lymphoblastic leukemia. J Pediatr Hematol Oncol 32: e323-e330, 2010.

20. Gou H, Zhou J, Ye Y, Hu X, Shang M, Zhang J, Zhao Z, Peng W, Zhou Y, Zhou Y, et al: The prevalence and clinical profles of FLT3-ITD, FLT3-TKD, NPM1, C-KIT, DNMT3A, and CEBPA mutations in a cohort of patients with de novo acute myeloid leukemia from southwest China. Tumour Biol 37: 7357-7370, 2016.

21. Bennett JM, Catovsky D, Daniel MT, Flandrin G, Galton DA, Gralnick HR and Sultan C: Proposals for the classification of the acute leukaemias. French-American-British (FAB) co-operative group. Br J Haematol 33: 451-458, 1976.

22. Creutzig U, van den Heuvel-Eibrink MM, Gibson B, Dworzak MN, Adachi S, de Bont E, Harbott J, Hasle H, Johnston D, Kinoshita A, et al: Diagnosis and management of acute myeloid leukemia in children and adolescents: Recommendations from an international expert panel. Blood 120: 3187-3205, 2012. 
23. Van Limbergen H, Poppe B, Michaux L, Herens C, Brown J, Noens L, Berneman Z, De Bock R, De Paepe A and Speleman F: Identification of cytogenetic subclasses and recurring chromosomal aberrations in AML and MDS with complex karyotypes using M-FISH. Genes Chromosomes Cancer 33: 60-72, 2002.

24. Meyer C, Hofmann J, Burmeister T, Gröger D, Park TS, Emerenciano M, Pombo de Oliveira M, Renneville A, Villarese P, Macintyre E, et al: The MLL recombinome of acute leukemias in 2013. Leukemia 27: 2165-2176, 2013.

25. Reimer J, Knöß S, Labuhn M, Charpentier EM, Göhring G, Schlegelberger B,Klusmann JHandHecklD: CRISPR-Cas9-induced $\mathrm{t}(11 ; 19) /$ MLL-ENL translocations initiate leukemia in human hematopoietic progenitor cells in vivo. Haematologica 102: 1558-1566, 2017.

26. Steckel NK, Groth C, Mikesch JH, Trenschel R, Ottinger H, Kordelas L, Mueller-Tidow C, Schliemann C, Reicherts C, Albring JC, et al: High-dose melphalan-based sequential conditioning chemotherapy followed by allogeneic haematopoietic stem cell transplantation in adult patients with relapsed or refractory acute myeloid leukaemia. Br J Haematol 180: 840-853, 2018

27. Cui L, Liu Y, Pang Y, Qian T, Quan L, Cheng Z, Dai Y, Ye X, Pang Y, Shi J, et al: Emerging agents and regimens for treatment of relapsed and refractory acute myeloid leukemia. Cancer Gene Ther 27: 1-14, 2020 .

28. Westhus J, Noppeney R, Dührsen U and Hanoun M: FLAG salvage therapy combined with idarubicin in relapsed/refractory acute myeloid leukemia. Leuk Lymphoma 60: 1014-1022, 2019.

29. Wang L, Xu J, Tian X, Lv T and Yuan G: Analysis of efficacy and prognostic factors of CLAG treatment in Chinese patients with refractory or relapsed acute myeloid leukemia. Acta Haematol 141: 43-53, 2019.
30. Bao Y, Zhao J and Li ZZ: Comparison of clinical remission and survival between CLAG and FLAG induction chemotherapy in patients with refractory or relapsed acute myeloid leukemia: A prospective cohort study. Clin Transl Oncol 20: 870-880, 2018.

31. Amadori S, Arcese W, Isacchi G, Meloni G, Petti MC, Monarca B, Testi AM and Mandelli F: Mitoxantrone, etoposide, and intermediate-dose cytarabine: An effective and tolerable regimen for the treatment of refractory acute myeloid leukemia. J Clin Oncol 9: 1210-1214, 1991.

32. Ringden O, Labopin M, Ehninger G, Niederwieser D, Olsson R, Basara N, Finke J, Schwerdtfeger R, Eder M, Bunjes D, et al: Reduced intensity conditioning compared with myeloablative conditioning using unrelated donor transplants in patients with acute myeloid leukemia. J Clin Oncol 27: 4570-4577, 2009.

33. Luger SM, Ringden O, Zhang MJ, Perez WS, Bishop MR, Bornhauser M, Bredeson CN, Cairo MS, Copelan EA, Gale RP, et al: Similar outcomes using myeloablative vs. reduced-intensity allogeneic transplant preparative regimens for AML or MDS. Bone Marrow Transplant 47: 203-211, 2012.

34. Wang L, Devillier R, Wan M, Decroocq J, Tian L, Fürst S, Wang LN, Vey N, Fan X, Blaise D and Hu J: Clinical outcome of FLAG-IDA chemotherapy sequential with Flu-Bu3 conditioning regimen in patients with refractory AML: A parallel study from Shanghai Institute of Hematology and Institut Paoli-Calmettes. Bone Marrow Transplant 54: 458-464, 2019. International (CC BY-NC-ND 4.0) License. 